\title{
Industry 4.0-Oriented Chipless RFID Backscatter Signal Variable Polarization Amplitude Deep Learning Coding
}

\author{
Guolong Shi $\mathbb{D},{ }^{1,2}$ Yigang $\mathrm{He}^{1}{ }^{1}$ Lichuan $\mathrm{Gu}^{2}$ and Jun $\mathrm{Jiao}^{2}$ \\ ${ }^{1}$ School of Electrical Engineering and Automation, Wuhan University, Wuhan, Hubei 430072, China \\ ${ }^{2}$ School of Information and Computer, Anhui Agricultural University, Hefei, Anhui 230036, China \\ Correspondence should be addressed to Guolong Shi; shiguolong@whu.edu.cn
}

Received 10 August 2021; Revised 31 August 2021; Accepted 1 September 2021; Published 24 September 2021

Academic Editor: Yuanpeng Zhang

Copyright ( 2021 Guolong Shi et al. This is an open access article distributed under the Creative Commons Attribution License, which permits unrestricted use, distribution, and reproduction in any medium, provided the original work is properly cited.

\begin{abstract}
Due to the weak network security protection capabilities of control system network protocols under Industry 4.0, the research on industrial control network intrusion detection is still in its infancy. This article discussed and researched the intrusion prevention technology of industrial control networks based on deep learning. According to the electromagnetic scattering theory, the backscatter signal model of the chipless tag was established as a chipless tag structure. Polarized deep learning coding was used for the label; that was, deep learning coding was performed on the copolarization component and the cross-polarization component at the same time, and a 16-bit deep learning coding bit number was obtained. The wave crest deep learning coding was used for the split ellipse ring patch label, and the 6-bit deep learning coding bit number was obtained. Then, the poles of the scattered signal of the tag were extracted to identify the tag. The variable polarization effect was achieved by adopting the dipole resonant unit with the two ends bent. Aiming at the problem of low detection rate caused by the shallow selection of feature classification of intrusion prevention systems, an industrial control network intrusion prevention model based on selfdeep learning encoders and extreme learning machines was proposed to extract features from industrial control network data through deep learning. For accurate classification, the theoretical judgment was also verified through simulation experiments, and it was proved that the detection rate of the model has also improved. It forms a set of industrial control network intrusion prevention system with complete functions and superior performance with data acquisition module, system log module, defense response module, central control module, etc. The matrix beam algorithm was used to extract the poles and residues for the late response, and the extracted poles and residues were used to reconstruct the signal. The reconstructed signal was compared with the scattered signal to verify the correctness of the pole extraction. Finally, the tags were processed and tested in the actual environment, and the measured results were consistent with the theoretical analysis and simulation results.
\end{abstract}

\section{Introduction}

"Industry $4.0 "$ is an era in which information technology promotes industrial transformation. At the same time, the concept of "Internet + " has triggered a major integration and transformation of information technology and the industry [1]. Therefore, the extensive application of IT technology in industrial control systems has transformed it from a proprietary and closed system into a highly open and interconnected system. While work is convenient and production efficiency is improved, the networked development of industrial control systems has led to system safety in case of that risks and threats of intrusion continue to increase [2]. Chip- less tags do not require silicon chips, so their production costs are relatively low compared to chip tags. Compared with traditional automatic identification technologies such as bar codes, two-dimensional codes, and card recognition, RFID technology has an absolute advantage [3]. As an emerging automatic identification technology, frequency identification has the characteristics of small size, large capacity, long life, and reusability. This technology can be combined with Internet, communication, and other technologies to realize the tracking and information of items on a global scale by M. R. Souryal et al. [4]. With the continuous development of this technology, its application field is expanding day by day, and it has become a current hot 
research field with a wide range of application prospects. The cost of labels is a major bottleneck in the application and development of technology. Only by reducing the cost of labels can it be widely used [5]. The design and research of the frequency band chipless or label is based on this background. Because there is no microchip and on-chip power supply, the chipless arsenic label greatly reduces the cost. The smart card has friction with the reader terminal every time it is used, which is easy to wear. The RFID tag and the reader terminal communicate wirelessly, there is no friction, and the RFID tag can be encapsulated. Inside the shell, there is no direct contact with the outside world, and it will not affect the recognition effect, so the RFID tag is more durable [6].

Low-cost chipless radio frequency tags have the most potential to become a substitute for barcodes, so chipless tags have become a hot spot in current research. At present, RFID tags widely used in the commercial market are mainly divided into active tags and passive tags with integrated silicon chips. These two types of tags have a high cost of mass production, which limits the popularization and development of RFID technology [7]. Therefore, the concept of chipless RFID tags with low cost advantages is proposed.

Based on the in-depth understanding of the importance of polarization information, variable polarization technology has begun to be used correspondingly in stealth, antistealth and interference, and antijamming [8]. With the further development of technology, it has put forward higher requirements for the polarization system: small size, low power consumption, and good antioverload performance. Therefore, this paper takes the research of proximity detection system RFID as the background and mainly focuses on the difficulty of miniaturization of variable polarization RFID in variable polarization system [9]. The chipless tag based on phase deep learning coding is composed of three square microstrip patch RFIDs, and each RFID is loaded with an open-circuit microstrip transmission line. The three RFIDs have adjacent resonant frequencies. When excited by their respective resonant frequencies, they reflect backscattered signals with different phase characteristics, which completes the phase modulation of the backscattered signals [10]. Since the symbol period of the tag is controlled by the frequency of the system oscillator, shortening the symbol period requires increasing the oscillator frequency. The higher the oscillator frequency, the greater the energy consumption. It is not wise to increase the data rate by increasing the oscillator frequency. Aiming at the problem of low detection rate caused by the shallow selection of feature classification of intrusion prevention systems, an industrial control network intrusion prevention model based on self-deep learning encoders and extreme learning machines is proposed to extract features from industrial control network data through deep learning. For accurate classification, the theoretical judgment is also verified by simulation experiments, and it is proved that the detection rate of the model has also improved [11]. According to the different phase characteristics of the backscatter signal, each tag can be designed as a unique and immutable chipless tag design. In addition to the deep learning coding capacity and size of the tag, whether the tag can be realized in the actual environ- ment which is also very important. Since depolarizing technology can improve the robustness of tag detection, the research on variable polarization chipless RFID tags has important application value and practical significance [12].

\section{Related Work}

Low-cost chipless radio frequency tags have the most potential to become a bar code substitute. Experts and scholars have done a lot of work and designed many types of chipless tags. The label design methods are also different. They are classified according to the deep learning coding method and are roughly divided into the following four categories [13]: based on time-domain delayed reflection, the typical label structure is based on surface acoustic wave (SAW) for the spectral characteristics, and its typical tag structure is a chipless tag with a multispiral resonator. Based on the amplitude/phase modulation of the reflected signal, its typical tag structure is a chipless tag. For the conductor complex natural resonance frequency, the principle of this type of label is that the scattered signal of the label carries the structural information of the label, and the purpose of identifying the label is achieved through the analysis of the scattered signal. At present, many types of chipless RFID tags have been designed based on the above four methods. Among them, tags are designed based on frequency domain characteristics, and the complex natural resonance frequency of conductors is more common [14]. So far, many domestic and foreign experts, scholars, and scientific research teams have conducted in-depth research on chipless RFID. These scientific research teams have put forward a variety of chipless RFID tag design ideas, hoping to solve the current chipless RFID tag design from different angles. The size, deep learning coding capacity, and label detection problems are faced by the Internet. Garbati et al. [15] divided chipless RFID tags into the following types according to the different ways of deep learning coding of data information: based on time domain deep learning coding and frequency domain deep learning coding, early polarization studies of type and other types began in the field of radar polarization in the early 1990s. Among them, Bekkali et al. [16] proposed a threeparameter trajectory method. Decarli and Dardari [17] proposed the concept of optimal target polarization, pointing out that there are several polarization states corresponding to the maximum and minimum energy of the radar receiving signal. He proposed the zero-polarization description method, established the basic theory of radar polarization, and became the founder of radar polarization research.

The surface acoustic wave is reflected by the reflector group for deep learning coding, and the reflected surface acoustic wave is emitted through the fork energy converter to generate electromagnetic waves. The RFID tag has a range of up to several meters, can identify high-speed moving targets, and has strong anti-interference performance. However, the fork energy converter is expensive, and the reflector group is large in size and cannot be bent. Aliasgari et al. [18] used two-way cross-energy converters and $Z$-shaped path reflector groups to greatly reduce the volume of RFID tags. Passive tags based on multiresonators generally consist of 
one receiving RFID, one resonant circuit, and one transmitting RFID sister. It needs to transmit a pulse signal with a wide frequency spectrum. In the resonant circuit, each resonant unit is a bit, which receives RFID. The received pulse signal goes through the resonant circuit for deep learning coding and then is transmitted by the RFID transmitter. The label designed by the feeding method can be completely printed, and the performance is stable. Babaeian and Karmakar [19] designed a 35-bit chipless RFID tag, which has a larger capacity, but the circuit is complicated, and the disadvantages of larger size still exist. Guidi et al. [20] designed a 3-bit chipless RFID tag with 3 patch units with close resonant frequencies and a rate close to the open-circuit highsniper line. Changing the length of the high-resistance line is equivalent to changing the load of the chip RFID. Thus, affecting the phase of the backscattered signal, so the deep learning coding capacity is small, and the size is too large. Some scholars have designed a circular patch label with a circular slit. The radius of the circular slit has a one-to-one correspondence with the wavelength of the resonant frequency of the backscatter signal, so this kind of label has many advantages such as large deep learning coding capacity and small size, but the structure is relatively complex [21]. The horizontal slot length of the label patch corresponds to the civil resonance frequency of horizontally polarized electromagnetic waves, and the vertical slot length of the label patch corresponds to the RFID resonance frequency of vertically polarized electromagnetic waves.

This article introduces the basic theory of polarization state representation and elliptical polarization synthesis and discusses and summarizes some methods of polarization parameter measurement. Based on these theoretical knowledge, the principles of receiving and transmitting in the variable polarization system are further discussed, and the basic implementation scheme of the miniaturized variable polarization system is given. Finally, based on the miniaturization requirements of the variable polarization system, an important part of the system, a double-end-fed square microstrip dual-polarization RFID, is simulated and designed. Based on the principle of backscatter modulation, a method based on the polarization characteristics of electromagnetic waves is proposed. Chipless RFID system with variable polarization technology and the indicators of the system and each component are given. The system has the advantages of large deep learning coding capacity, simple structure, and low cost. And its variable polarization characteristics are simulated, and the results ideally verify that the active variable polarization system can use dual-end-fed dual-polarized microstrip RFID as an orthogonal dual-fed RFID to achieve the composite output of various polarized waves.

\section{Design of a Chipless RFID System with Variable Polarization of Backscattered Signal and Variable Polarization for Industry 4.0- Oriented Amplitude Deep Learning Coding}

3.1. RFID Performance Mechanism. RFID is the main component of the radio frequency system. For the identification system, RFID should have a simple structure and low cost. The main radio frequency RFIDs currently used include monopole RFID, dipole RFID, and microstrip RFID. Figure 1 shows the hierarchical distribution of RFID performance mechanisms.

For RFID in the wireless communication system, the working principle follows the electromagnetic field theory; through the alternating electromagnetic field to form an electromagnetic wave to transmit information, the electromagnetic field propagation under ideal conditions satisfies Maxwell's equation.

$$
\begin{aligned}
& \nabla \times E+j \omega \alpha \times H=0, \\
& \nabla \times H+j \omega \alpha \times E=J .
\end{aligned}
$$

It can be seen from the above formula that the propagation direction of the electromagnetic field is consistent with the direction of the energy flow density. According to the electromagnetic field theory, the electromagnetic field area which was emitted by RFID in the radio frequency identification system can be divided into three parts: induction near field, radiation near field, and far field. The induction near field is the area close to the radio RFID and dominated by the induction field. The main form of energy storage is stored; the box radio near field is mainly dominated by the radio field, and the radiation angle is related to the distance of the RFID. The energy in the far field of the box is mainly emitted by electromagnetic waves, and the radiation angle has nothing to do with the distance. The RFID in this article belongs to ultrahigh frequency RFID, which mainly works in the far field of radiation.

$$
E=i d l / 4 \pi \times \cos \alpha \times \exp (-j w)
$$

RFID is a device for sending and receiving signals in a radio frequency identification system. The performance of RFID will affect the identification of tags. This section mainly introduces several main performance parameters of RFID. The relationship between RFID's box shootability and space coordinates is called the RFID directional function, and the national shape drawn according to the functional relationship is called the directional diagram. For the radiation characteristics of RFID, the directivity function is defined as:

$$
\iint L / 4 \pi r^{2} \times \sin \alpha \cos \beta \times d \alpha d \beta=H .
$$

Among them, $r$ is the radiation power, $L$ is the input power, and $H$ is the total power consumed in the RFID conductor and the dielectric plate. If the power consumed by RFID is regarded as the loss of resistance, so:

$$
\left\{\begin{array}{l}
S_{x}=A x a^{2} / 4 \pi \times P(\operatorname{tag}), \\
S_{y}=A y b^{2} / 4 \pi \times G(\operatorname{tag}) .
\end{array}\right.
$$

Therefore, the relationship between the efficiency of RFID and the radiation resistance and loss resistance can 


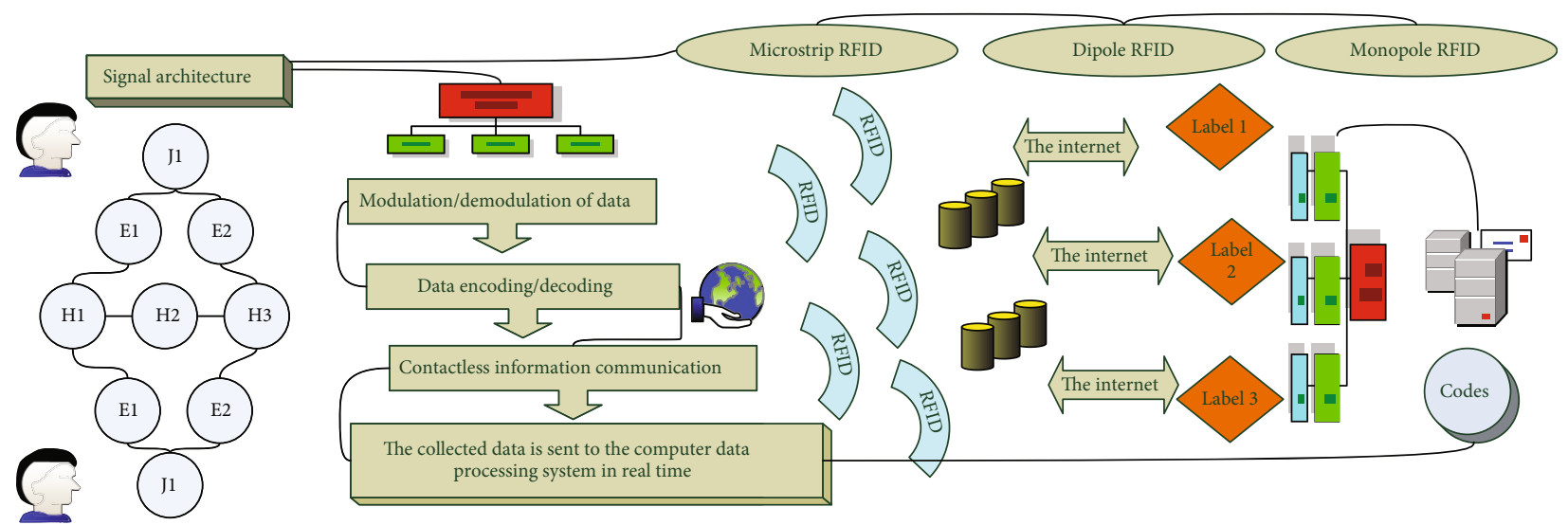

FIGURE 1: Hierarchical distribution of RFID performance mechanism.

be obtained. Therefore, the RFID efficiency can be improved by reducing the loss resistance and increasing the box emission resistance. The input impedance of RFID is related to the shape, size, and excitation method of RFID and the distance between surrounding objects. RFID is connected to the feeder, and impedance matching is usually required. When the input impedance of RFID is pure resistance and the characteristic impedance of the feeder is equal, no power reflection is the best state at this time.

$$
I=\left[\begin{array}{ccc}
i \cos \alpha \sin \beta & 0 & 0 \\
0 & i \sin \alpha \cos \beta & 0 \\
0 & 0 & i \cos \alpha \cos \beta
\end{array}\right] .
$$

RFID polarization represented the trajectory of the end point of the space electric field vector. The polarization mode of RFID can be divided into $H$ kinds of linear polarization, circular polarization, and column circular polarization. Since the length of a monopole is about a quarter of a wavelength and its working frequency is determined by the size of its monopole, RFID of various working frequency bands can be made as needed.

$$
\begin{array}{r}
\frac{w_{r}+1}{2}+\frac{w_{r}-1}{2} \times \frac{w_{r}^{2}}{a}=W, \\
\frac{E^{\prime}}{H^{\prime}}=\left|\frac{W_{1}-W^{*}{ }_{a}}{W_{1}+W_{a}^{*}}\right|^{2} \times \exp (-j w) .
\end{array}
$$

For the shape of the RFID box radiation pattern, it is omnidirectional on the $H$ side; it is a figure eight on the $E$ side. For the RFID feeding method, the input impedance of this type of RFID is close to $50 \mathrm{ohms}$, which matches the $50 \mathrm{ohms}$ feeder line. Therefore, microstrip lines, coaxial cables, coplanar waveguides, etc., can be used for feeding. The various performances of single-pole RFID make it applicable to RFID systems.

3.2. Variable Polarization Backscatter Modulation. The backscatter modulation terminal is responsible for processing the received signal and reading the characteristic infor- mation of the object. It is composed of middleware and a background processing network. The middleware is responsible for communicating with the reader, obtaining the information from the reader and performing corresponding processing, and at the same time transmitting the information to the back-end network for processing, and finally obtaining the characteristic information of the object. The background processing network generally includes modules of data center, control center, and network equipment. The data must be used to store the characteristic data of the item. After receiving the information from the reader, the background network will query the database according to the item ID to determine the characteristic data of the item. Generally speaking, the close staring system is mostly realized by quasi-static field collision. Figure 2 shows the variable polarization backscatter modulation mechanism. As mentioned earlier, the energy exchange mode between the reader and the tag at this time is similar to the transformer model, that is, the load modulation mode. This method actually changes the voltage on the reader's antenna by changing the on and off of the load resistance on the tag antenna, so as to realize the amplitude modulation of the antenna voltage by the short-distance tag. This modulation method is widely used in the control of system and mainly controls the background processing network to ensure normal communication.

For the staring system of $2 \mathrm{GHz}$ or higher frequency, it works in the typical far field. The energy transfer method between the reader and the tag can only be backscatter modulation. Backscatter modulation refers to the communication method used when the electronic tag in the passive staring system sends data to the reader. The tag generally contains an antenna and a chip. An impedance switch is used in the chip to adjust the input impedance of the chip itself. Since the data type of the industrial control network intrusion detection data set is a mixture of continuous and discrete data, it is necessary to perform preprocessing such as normalization of the data. The sparse autoencoder can make the preprocessed data more sparse after feature extraction. The feature data with high sparsity after dimensionality reduction is used as the input of the classifier, and then, the classifier is used to classify the feature data. Assuming that 


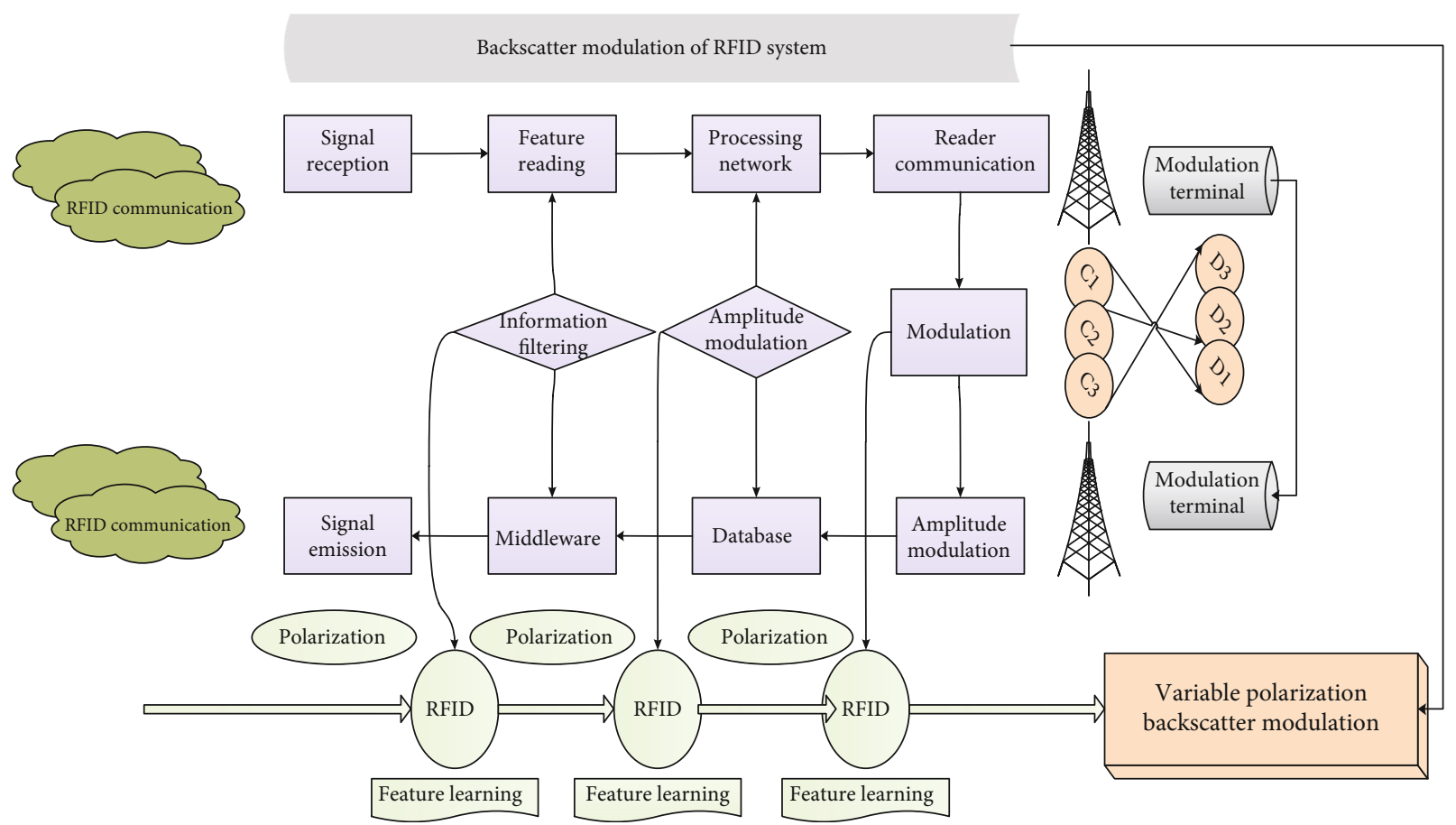

Figure 2: Variable polarization backscatter modulation mechanism.

the data signal to be sent is a signal with two levels, it is modulated by a logic gate and an intermediate frequency signal, and the modulated signal is controlled. The opening and closing of the impedance switch change the input impedance of the chip, that is, changes the impedance matching between the chip and the antenna. When the tag antenna is conjugated to the connected tag chip, most of the energy received by the antenna will be transferred to the chip, and the energy reflected back to the reader is small. The reflected echo is modulated in a method similar to RFID modulation.

3.3. Amplitude Deep Learning Coding Optimization. Since the deep learning coding angle of the linear slot label is related to the direction of the excitation electric field, the strip gap in the linear slot label structure is changed to a shape, showing a relationship where the deep learning coding angle is not related to the direction of the excitation electric field. Figure 3 shows a schematic diagram of amplitude deep learning coding optimization. The $V$-shaped structure contains two slit arms $A$ and $B$ of equal size, and the size of the two arms is electrode. The fixed $\operatorname{arm} A$ is placed in the horizontal direction, and the angle between the two arms is 0 for deep learning to encode data. The horizontally polarized plane electromagnetic wave with electric field intensity enters the new label vertically. It can be seen that the current flows are two circuits around the gap. The formed gap label is used as a standard label, and the scattered electric field component of arm $A$ in the horizontal direction is $E$. The value of the electric field component in the horizontal direction and the component value in the vertical direction of the scattered electric field around the arm $B$ are obtained, respectively.
According to the distribution of the frequency spectrum when the label is working, we select 16 angles with an interval of 10 for the deep learning coding address information, corresponding to the 4-bit address information, and the deep learning coding capacity is 4 bit. Substituting the electric field value of the label at the working frequency into the formula, the recognition result is shown in paper.

It can be seen that all addresses can be distinguished correctly, and the angle recognition error is less than 2.24. And different deep learning coding states all occupy the same frequency band range of $2.92 \mathrm{GHz}-4.28 \mathrm{GHz}$. Figure 4 shows the broken line graph of the amplitude information of the deep learning encoding. In passive systems with frequency bands or higher frequencies, the electromagnetic waves emitted by the reader are reflected to complete the data transmission from the tag to the reader.

The radio frequency signal generated by the reader is radiated to the space by the antenna. Assuming that the gain of the reader's transmitting antenna is taste, the transmitting power is the effective radiation power of the antenna, which is the product of the reader's transmitting power and the antenna gain. The pooling layer can effectively reduce the size of the matrix by reducing the length, width, and depth of the matrix, thereby reducing the parameters in the subsequent fully connected layer and at the same time making feature sparse can accelerate the calculation speed and prevent overfitting. The forward propagation of the pooling layer is completed by performing a maximum or average operation on the movement of the pooling core. According to radar technology, an object whose volume exceeds half of the electromagnetic wavelength can be reflected, and the energy reflected by the tag is proportional to the reflective crosssectional area of the object. In addition, considering the 


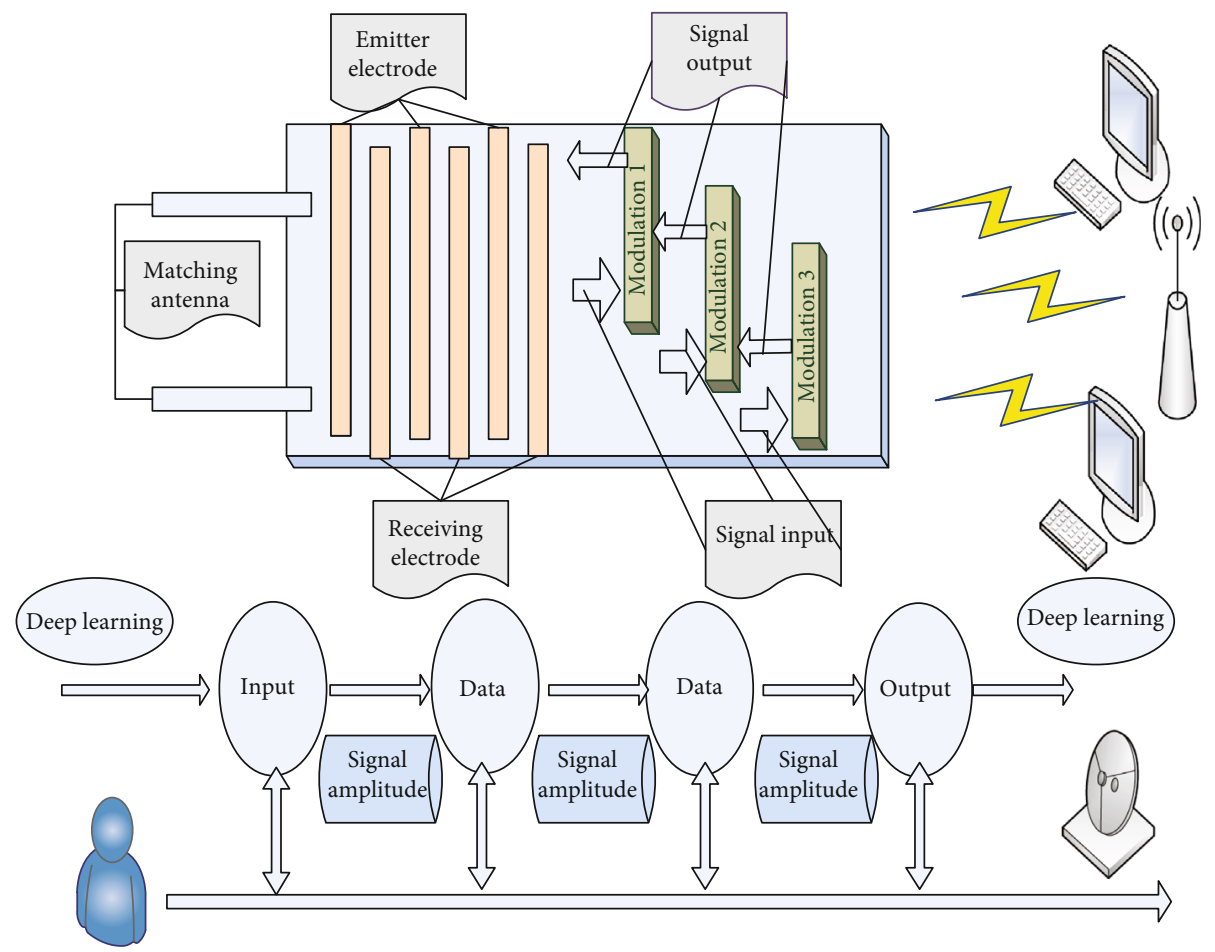

Figure 3: Schematic diagram of amplitude deep learning coding optimization.

impedance matching between the tag chip and the tag antenna, the impedance matching coefficient of the tag is defined as $q$. Considering the limit situation, when the tag chip and the antenna impedance match, all the energy is transmitted from the tag antenna to the inside of the chip, and the tag reflects the power. When the tag chip and the antenna impedance do not match, all the energy is reflected back to the reader, and the system uses this backscatter modulation method to return the signal to the reader.

\section{Industry 4.0-Oriented Amplitude Deep Learning Coding Backscatter Signal Variable Polarization Chipless RFID System Application and Analysis}

4.1. Backscatter Signal Preprocessing. In this paper, the resonant frequencies of the three antenna units are designed as $f 1, f 2$, and $f 3$, which are all between 2.1 and $2.5 \mathrm{GHz}$, and the frequency separation between each other is about five stones. The reader in this system has already learned these three frequencies. When the system is working, the reader transmits the frequency of five multifrequency access signals. Here is a simplified model, and the multifrequency access signal is set as a sine wave. The three antenna elements in the chipless tag are excited by their respective resonant frequency waves and reflect backscattered signals with different phase characteristics. The signals with phase characteristics returned by simulation can be obtained. After fixing the phase, we can get three discrete phase values. If each patch unit can be designed to make $n$ different situations of its own resonant frequency wave in the reflected signal, then

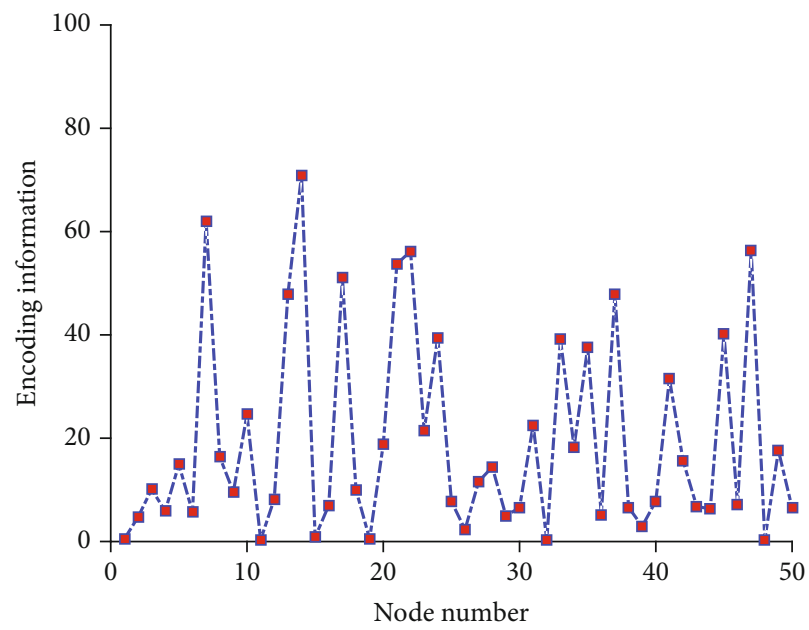

FIgURE 4: The broken line graph of the amplitude information of the deep learning coding.

the chipless tags composed of three patch units have different combinations of minerals. Figure 5 shows the flow chart of the backscatter signal preprocessing. According to the different phase characteristics of the backscattered signal, each tag can be designed to be unique and immutable.

Here, we choose a material with a dielectric constant of $a$ and a thickness of $b$. The working frequency band is between one. According to the design method of the rectangular microstrip antenna introduced above, the side length of the square microstrip patch is initially selected. Without loading any transmission lines, we design the antenna to match its impedance. In this section, commercial electromagnetic simulation software will be used to obtain the parameter values 


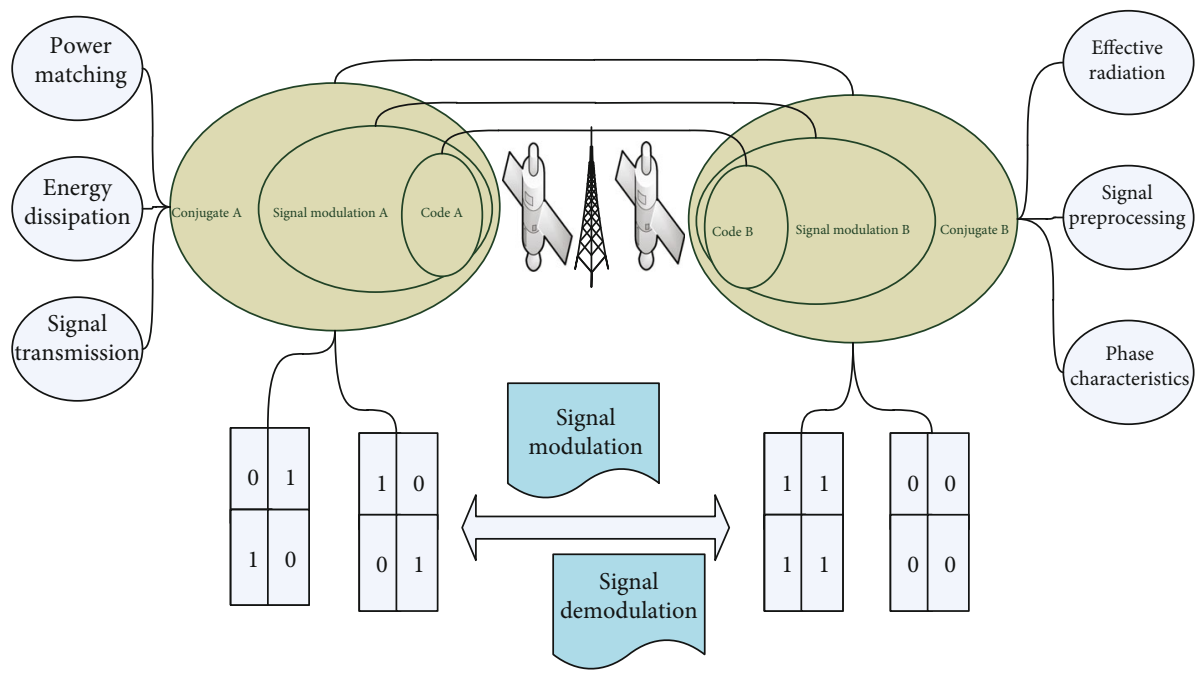

FIGURE 5: Flow chart of backscatter signal preprocessing.

of the optimized square microstrip patch antenna. The characteristic impedance of the embedded microstrip feeder and the width of the feeder are one. The size of the parameters $a$ and $b$ can be adjusted to optimize the antenna to match. In the antenna reflection coefficient with the open end of the embedded microstrip feeder as the antenna input port, it can be seen that the antenna is well matched to $50 \mathrm{ohms}$. Here, we use three square microstrip units to form an antenna array. The three square microstrip patch antenna units have different resonant frequencies, and the resonant frequencies are adjacent, all within a range. We select the appropriate side length according to the design theory of the microstrip patch antenna and the operating frequency requirements.

4.2. Simulation of Chipless RFID System. The measurement process is carried out in the actual environment rather than in the microwave anechoic chamber. The measuring instrument is a N5230A vector network analyzer with a transmitting power of $3 \mathrm{~dB}$. Two identical standard butterfly antennas are used as the transmitting and receiving antennas of the reader. We connected the two identical standard antennas before measurement. On port 1 and port 2 of the network analyzer, they were used as transmitting and receiving antennas, respectively. The antenna works in the frequency range of $1.572-3.714 \mathrm{GHz}$. We placed the two antennas horizontally and vertically and placed a device that can fix the sensor at a distance of $0.8 \mathrm{~m}$ from the antenna and placed the device in the middle of the two antennas. It could be seen that the sensor was at 50 . Through random selection, 5000 sets of data in the data set are used as the training set, and 1000 sets of data are used as the test set. The sigmoid function is used as the activation function of the sparse autoencoder, the sparsity parameter is 0.25 , the number of hidden layers is $n=14$, and the number of cycles is 50. The extreme learning machine as the classification module also uses the sigmoid activation function. After many experiments, the input weight, the number of nodes, the bias of the hidden unit, and other parameters are deter- mined. The working frequency was the same, and the working frequency band was relatively narrow, which could effectively improve the high spectrum utilization rate. We put the values of the resonance frequency into the formula and saw the article for the recognition results of angle and underresistance. It could be seen that the recognition errors of angles were all less than 3, and the recognition errors of resistors were all less than 10 , so the sensor could realize the correct identification of angle 0 and resistance value.

The sensor had two resonant frequencies, and the resonant frequency was only related to the length of the scattering unit and did not change with the angle. Figure 6 shows the amplitude response curve of the resonant frequency of the chipless RFID. It could be seen from the figure that at the resonance frequencies of $15 \mathrm{~Hz}$ and $95 \mathrm{~Hz}$, the scattered electric fields in the horizontal and vertical directions were pulled, respectively. Bringing the parameters into the formula, the recognition angles were 91.5 and 101.5, respectively. It could be obtained that the angle errors are 1.5, $1.5,0.9$, and 4 .

4.3. Example Application and Analysis. The plane wave is incident on the sensor vertically, and the two resonance frequencies are obtained by simulation. At the resonance frequency, the horizontal and vertical backscattered electric fields are E1, E2, H1, and $\mathrm{H} 2$. Since the angle recognition formulas of two scattering units and one scattering unit are the same, $W$ incorporates the simulated backscattering electric field value into the formula, and $W$ can realize the recognition of angle from the perspective of deep learning to encode address information. Figure 7 shows the identification curve of the tag angle information under the RFID backscattering electric field. The blue data line is the control group, which corresponds to the algorithm proposed in Reference [20], and the red data line is the experimental group, which corresponds to the algorithm in this paper. It can be seen that the red data line is smoother, which means that the algorithm in this paper is more superior. The respective resonant frequencies of the antenna are $f 1, f 2$, and $f 3$, and 


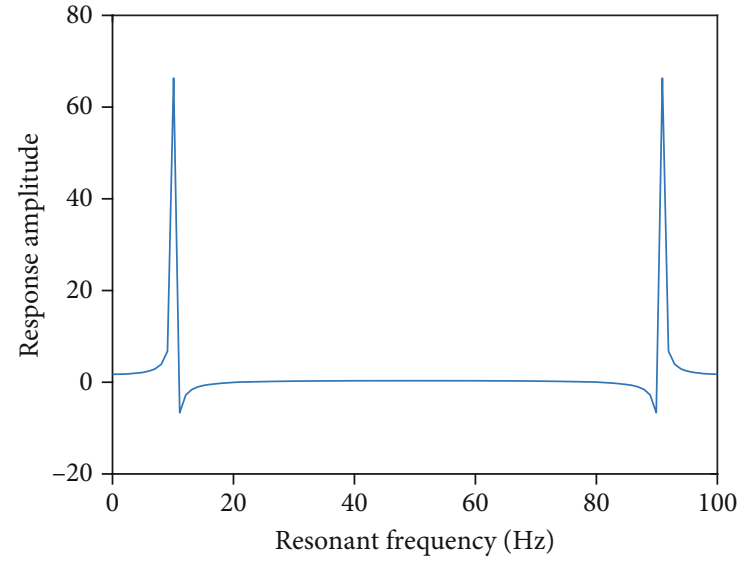

Figure 6: Amplitude response curve of resonant frequency of chipless RFID.

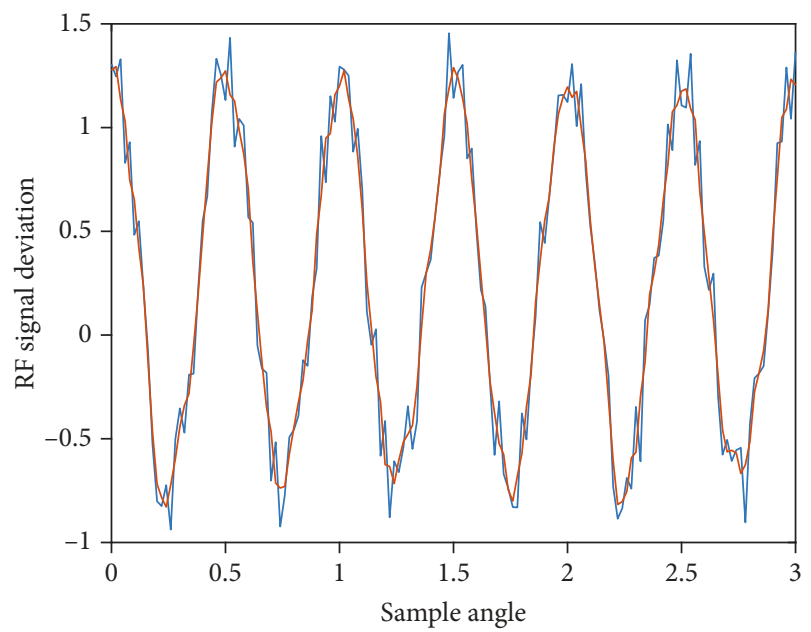

Figure 7: The identification curve of tag angle information under RFID backscattering electric field.

the return loss deviation value of each resonant point is lower than 1.5, which can be well matched. And it can be seen from the paper that the maximum mutual coupling between the three antennas is as low as $-20 \mathrm{~dB}$, which shows that the mutual coupling between the three antenna elements is small, and the mutual influence is within an acceptable range.

By measuring the horizontal and vertical components of the backscattered electric field, the identification of the tag's angle information is realized. The tag deep learning coding capacity can be up to 4 bit, the angle recognition error of the linear slot label is less than 1.55 , and the angle recognition error of the $V$-shaped slot tag is less than 2.24. The measurement result of the tag is consistent with the simulation result, and the measured resonance point is slightly smaller than $5 \mathrm{GHz}$. The designed RFID model has a simple structure and low cost. Different deep learning coding tags occupy the same frequency spectrum and occupy a narrow frequency band, which can reduce the cost of the entire radio frequency identification system. The designed tag can

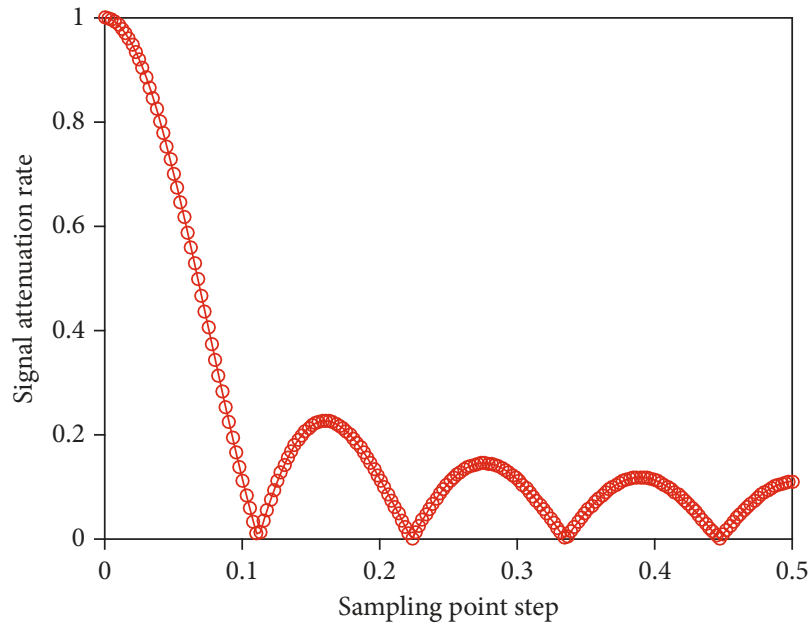

FIGURE 8: RFID polarization excitation signal gain rate depends on the sampling point of resonance frequency.

be applied to the Internet of Things as an angle sensor. When RFID frequency is low, the antenna feed mode is traditional coplanar waveguide feed; the antenna has two resonance points of $3.6 \mathrm{GHz}$. As it increases, the direction changes little, and it moves to the left. It is approximately equal to a quarter of the guided wave wavelength corresponding to 2 . Figure 8 shows the dependence of the RFID polarization excitation signal gain rate on the sampling point of the resonance frequency. For the entire antenna, the asymmetry of ACPW (asymmetric coplanar waveguide) causes the antenna to have four resonance points. By adjusting signal mode can be superimposed together to obtain a broadband antenna. However, due to the appearance of another butterfly-shaped gap, RFID attenuation rate will shift a little, and the signals merge into a resonant frequency.

The sensor is excited by linearly polarized waves, and the placement direction of the fixed arm $A$ is the same as the polarization direction of the excitation electromagnetic wave. That is, when the excitation electromagnetic wave is a horizontally polarized wave, the fixed $\operatorname{arm} A$ is placed in the horizontal direction. The results show that after the training set has been continuously increased the number of iterations, the improved sparse autoencoder reduces the loss value of the training set after dimensionality reduction and stabilizes at 0.1 . In other words, the training network is continuously converging, and the algorithm is extracted for the effect of the feature also tends to stabilize. When the wave is vertically polarized, the fixed arm $A$ is placed in the vertical direction. The antenna impedance and magnitude are different, which can be seen that the influence of $A$ is less than $1 / 3$. With the increase of phase, the degree of change in the imaginary part of impedance in the range of $0.01-0.1 \mathrm{rad} / \mathrm{s}$ is greater than that at $100-1000 \mathrm{rad} / \mathrm{s}$. When frequency is less than $0.1 \mathrm{rad} / \mathrm{s}$, the imaginary part of the impedance is lower than $50 \mathrm{~dB}$, and the real part is close to 50 .

Figure 9 shows the phase and amplitude information of the scattered electric field at different variable polarization frequencies. Using wireless radio frequency technology to read the sensor's scattered electric field information, we 


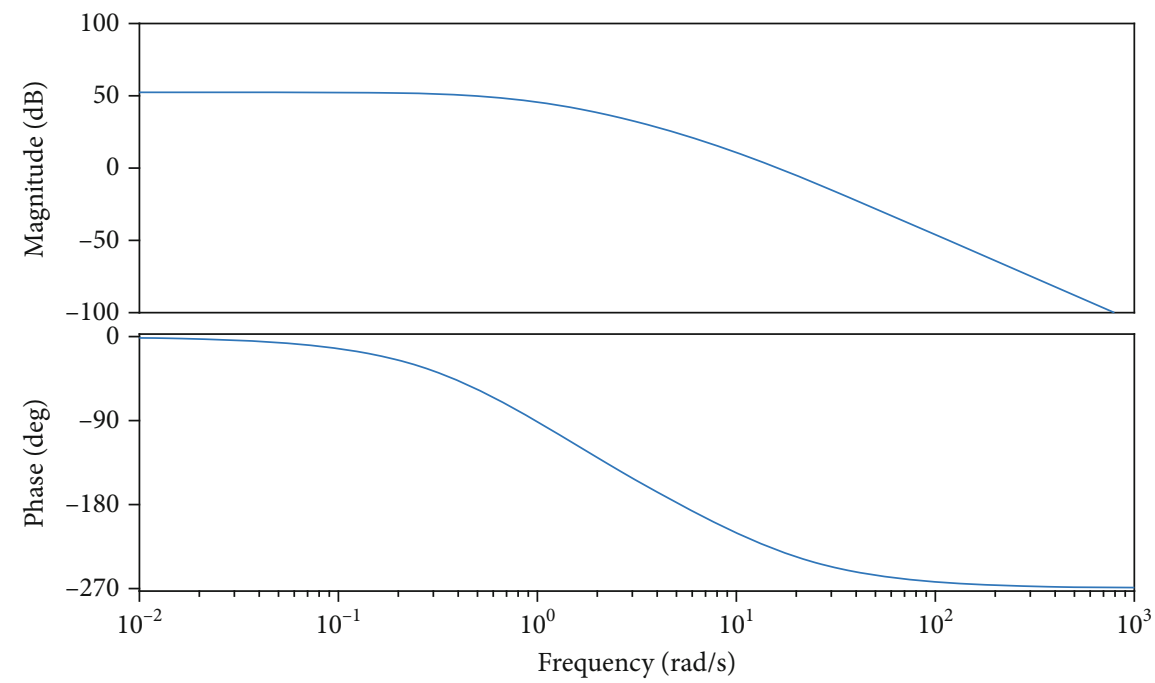

Figure 9: Scattered electric field phase amplitude information at different variable polarization frequencies.

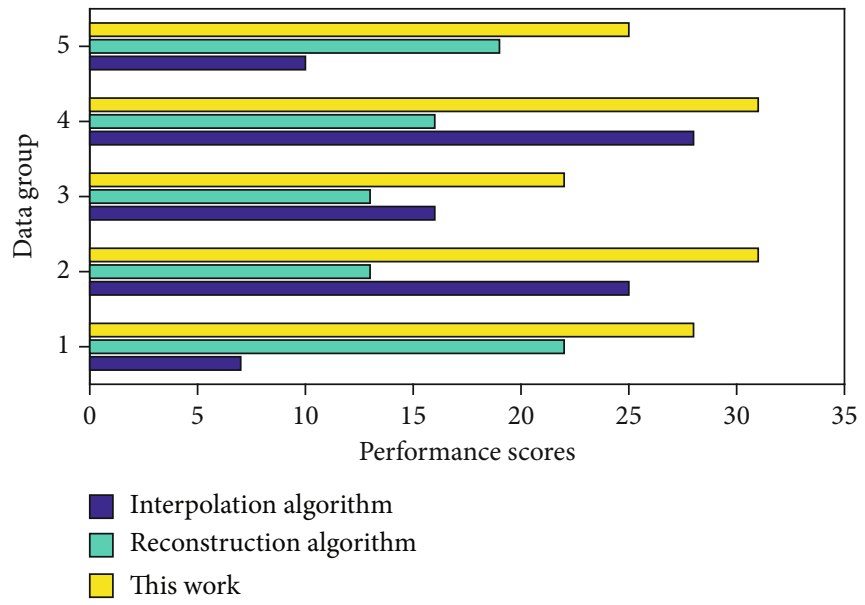

FIGURE 10: Histogram of score distribution of different coding algorithm models.

can realize the identification of the sensor's address information and the resistance to be measured. Through simulation, it can be known that the resonance frequency of the sensor is only related to the length of the box-fired patch, and the angle is only related to the backscattered electric field of the corresponding radiation unit. By measuring the horizontal and vertical backscattered electric fields, the angle and identification of resistance are given.

Figure 10 shows the histogram of the score distribution of different coding algorithm models. It can be seen from the histogram that the algorithm proposed in this paper has obtained relatively high scores in the 5 experimental groups and has excellent performance compared to the other two algorithms. The yellow bar graph represents the experimental group, and the other two colors of data represent the control group. Compared with interpolation algorithm and reconstruction algorithm, the algorithm designed in this paper performs better. For the resistance sensor with a single type unit, the angle recognition error is less than 3 , the resistance value recognition error is less than 1.5, and the deep learning coding capacity can reach 4 bit. For the resistance sensors of two units, the angle recognition error is less than 2.5, and the resistance recognition is less than 2.0. An RFID mode unit is added to realize the 5-bit deep learning coding capacity. In addition, the RFID sensor in this paper can be directly applied to the radio frequency identification system by changing the address information and resistance value to a wireless signal.

\section{Conclusion}

The components designed in this paper construct a chipless RFID field system based on the variable polarization scheme, and we use $2 \times 2$ array tag RFID and $4 \times 4$ array tag RFID to test and analyze the horizontal polarization branch and vertical polarization branch of the system separately. We compare the test results and use $4 \times 4$ array tag RFID to test and analyze the horizontal polarization branch and vertical polarization branch of the system at the same time. Combined with the measured experimental data, the specific 
process of the system identification tag RFID polarization mode is given, and the feasibility of the chipless RFID system based on variable polarization technology is verified. By lengthening and bending one of the feeding slots, an asymmetric coplanar waveguide feeding mode is formed, which improves the impedance matching of the antenna and increases the bandwidth of the antenna. At the same time, the $H$-angle loop conduction band is loaded in the butterfly slot, which improves the low-frequency gain of the antenna and makes the gain of the antenna in the working frequency band more flat. The gain within the bandwidth is all higher than $1.5 \mathrm{~dB}$, and the highest gain is $5.53 \mathrm{~dB}$; the simulation and actual measurement results are basically in agreement. The two resonance peaks in the cross-polarization direction are both used to determine the humidity change, which is beneficial to reduce the humidity detection error in practical applications. The two anomaly detection algorithms based on deep learning proposed in this paper are used as signal detection modules, which are combined with data acquisition module, system log module, defense response module, and central control module. Based on the above theoretical knowledge, an intrusion detection algorithm based on autoencoder and extreme learning is established. Through algorithm description and simulation experiments, it is proved that it meets the reliability requirements of "high detection rate and low false alarm rate" of industrial control network. A set of industrial control RFID network signal prevention system with complete functions and superior performance has been formed, and its effectiveness and reliability have been proved through system testing. In addition, the tag has variable polarization characteristics, which can improve the robustness of the sensor's detection in the actual environment.

\section{Data Availability}

The data used to support the findings of this study are included within the article.

\section{Conflicts of Interest}

The authors declare that they have no conflicts of interest.

\section{Acknowledgments}

This work was supported by the China Postdoctoral Fund General Project (No. 2021M692473) and the Natural Science Foundation of Anhui Province (2108085QF260), the National Natural Science Foundation of China Project: "Research on the Integrated Control Method of the Attachment Limit State of Agricultural Tracked Robot Based on Non-stationary Constraint" (No. 31671589), and the Provincial Science and Technology Major Special Project: "Research on Key Technologies for Precise Control of Healthy Pig Breeding and Intelligent Disease Early Warning in a Big Data Environment” (No. 201903a06020009).

\section{References}

[1] C. Feng, W. Zhang, L. Li, L. Han, X. Chen, and R. Ma, "Anglebased chipless RFID tag with high capacity and insensitivity to polarization," IEEE Transactions on Antennas and Propagation, vol. 63, no. 4, pp. 1789-1797, 2015.

[2] C. Boyer and S. Roy, "Coded QAM backscatter modulation for RFID," IEEE Transactions on Communications, vol. 60, no. 7, pp. 1925-1934, 2019.

[3] J. D. Griffin and G. D. Durgin, "Complete link budgets for backscatter-radio and RFID systems," IEEE Antennas and Propagation Magazine, vol. 51, no. 2, pp. 11-25, 2020.

[4] M. R. Souryal, D. R. Novotny, D. G. Kuester, J. R. Guerrieri, and K. A. Remley, "Impact of RF interference between a passive RFID system and a frequency hopping communications system in the $900 \mathrm{MHz}$ ISM band," IEEE Electromagnetic Compatibility Magazine, vol. 1, no. 3, pp. 97-102, 2012.

[5] U. Mc Carthy, G. Ayalew, F. Butler, K. McDonnell, and S. Ward, "The effects of item composition, tag inlay design, reader antenna polarization, power and transponder orientation on the dynamic coupling efficiency of backscatter ultrahigh frequency radio frequency identification," Packaging Technology and Science: An International Journal, vol. 22, no. 4, pp. 241-248, 2009.

[6] P. V. Nikitin, K. V. S. Rao, and LabVIEW-based UHF, "RFID tag test and measurement system," IEEE Transactions on Industrial Electronics, vol. 56, no. 7, pp. 2374-2381, 2020.

[7] J. Zhang, G. Y. Tian, A. M. J. Marindra, A. Sunny, and A. Zhao, "A review of passive RFID tag antenna-based sensors and systems for structural health monitoring applications," Sensors, vol. 17 , no. 2 , p. $265,2017$.

[8] C. Herrojo, F. Paredes, J. Mata-Contreras, and F. Martín, "Chipless-RFID: a review and recent developments," Sensors, vol. 19, no. 15, p. 3385, 2019.

[9] E. Denicke, H. Hartmann, N. Peitzmeier, and B. Geck, "Backscatter beamforming: a transponder for novel MIMO RFID transmission schemes," IEEE Journal of Radio Frequency Identification, vol. 2, no. 2, pp. 80-85, 2018.

[10] V. R. Sajitha, C. M. Nijas, T. K. Roshna, R. Vivek, K. Vasudevan, and P. Mohanan, "Polarization independent chipless RFID tag," Microwave and Optical Technology Letters, vol. 57, no. 8, pp. 1889-1894, 2015.

[11] C. Herrojo, J. Mata-Contreras, F. Paredes, A. Núñez, E. Ramon, and F. Martín, "Near-field chipless-RFID tags with sequential bit reading implemented in plastic substrates," Journal of Magnetism and Magnetic Materials, vol. 459, pp. 322$327,2018$.

[12] M. A. Islam and N. C. Karmakar, "Real-world implementation challenges of a novel dual-polarized compact printable chipless RFID tag," IEEE Transactions on Microwave Theory and Techniques, vol. 63, no. 12, pp. 4581-4591, 2020.

[13] M. Zomorrodi, N. C. Karmakar, and S. G. Bansal, "Introduction of electromagnetic image-based chipless RFID system," Sensor Networks and Information Processing, vol. 3, pp. 443448, 2019.

[14] V. Mulloni and M. Donelli, "Chipless RFID sensors for the Internet of Things: challenges and opportunities," Sensors, vol. 20, no. 7, p. 2135, 2020.

[15] M. Garbati, E. Perret, R. Siragusa, and C. Halope, "Ultrawideband chipless RFID: reader technology from SFCW to IRUWB," IEEE Microwave Magazine, vol. 20, no. 6, pp. 74-88, 2019. 
[16] A. Bekkali, S. Zou, A. Kadri, M. Crisp, and R. Penty, "Performance analysis of passive UHF RFID systems under cascaded fading channels and interference effects," IEEE Transactions on Wireless Communications, vol. 14, no. 3, pp. 1421-1433, 2015.

[17] N. Decarli and D. Dardari, "Time domain measurements of signals backscattered by wideband RFID tags," IEEE Transactions on Instrumentation and Measurement, vol. 67, no. 11, pp. 2548-2560, 2018.

[18] J. Aliasgari, M. Forouzandeh, and N. Karmakar, "Chipless RFID readers for frequency-coded tags: time-domain or frequency-domain?," IEEE Journal of Radio Frequency Identification, vol. 4, no. 2, pp. 146-158, 2020.

[19] F. Babaeian and N. C. Karmakar, "Hybrid chipless RFID tags-a pathway to EPC global standard," IEEE Access, vol. 6, pp. 67415-67426, 2018.

[20] F. Guidi, N. Decarli, D. Dardari, C. Roblin, and A. Sibille, "Performance of UWB backscatter modulation in multi-tag RFID scenario using experimental data," IEEE Ultra-Wideband, vol. 1, pp. 484-488, 2011.

[21] M. Forouzandeh and N. Karmakar, "Self-interference cancelation in frequency-domain chipless RFID readers," IEEE Transactions on Microwave Theory and Techniques, vol. 67, no. 5, pp. 1994-2009, 2019. 